C. Mizutani

Nagoya Math. J.

Vol. 87 (1982), 1-15

\title{
DEFINABILITY THEOREM FOR THE INTUITIONISTIC PREDICATE LOGIC WITH EQUALITY
}

\author{
CHIHARU MIZUTANI
}

\section{Introduction}

Svenonius' definability theorem and its generalizations to the infinitary logic $L_{\alpha_{1} \omega}$ or to a second order logic with countable conjunctions and disjunctions have been studied by Kochen [1], Motohashi [2], [3] or Harnik and Makkai [4], independently. In this paper, we consider a (Svenoniustype) definability theorem for the intuitionistic predicate logic $I L$ with equality.

First we recall Svenonius' theorem and Motohashi's theorem. Suppose that $L_{1}$ is a first order logic with equality, $L_{2}$ is a second order logic with countable conjunctions and disjunctions and $L$ is either $L_{1}$ or $L_{2}$. Let $P$ be a $k$-ary predicate constant not in $L, T(P)$ a set of sentences (resp. negative sentences) in $L_{1}(P)$ (resp. $L_{2}(P)$ ). In the case of $L=L_{2}$, we assume that the set of individual free variables are divided into two infinite disjoint sets $X$ and $Y$. Now, consider the following three conditions:

(i) For any models $\mathfrak{a}, \mathfrak{b}$ of $T(P), \mathfrak{a}|L=\mathfrak{b}| L$ and $\mathfrak{a} \cong \mathfrak{b}$ imply $\mathfrak{a}=\mathfrak{b}$.

(ii) $T(P) \vdash_{L(P)} \bigvee_{i=1}^{n}(\forall \bar{u})\left(P(\bar{u}) \equiv \varphi_{i}(\bar{u})\right)$ for some formulas $\varphi_{1}(\bar{x}), \cdots, \varphi_{n}(\bar{x})$ in $L$.

(iii) $T(P) \vdash_{L(P)}(\forall \bar{u})(P(\bar{u}) \equiv \varphi(\bar{u}))$ for some Motohashi P-formula $\varphi(\bar{x})$ in $L(P)$ whose free variables are among $\bar{x} \subseteq X$.

(See [3] or [4] about Motohashi $P$-formula in $L(P)$.)

Then, Svenonius' theorem is that the conditions (i) and (ii) are equivalent in the case of $L=L_{1}$ and Motohashi's theorem is that the conditions (i) and (iii), hence also (ii), are all equivalent in the case of $L=L_{2}$.

When we study the relations between these conditions for the intuitionistic predicate logic $I L$, we must consider the following syntactical condition (i)' instead of the semantical condition (i).

Received June 6, 1979. 
(i) $\operatorname{Isom}_{L}(I ; P, Q), T(P), T(Q) \vdash_{L(P, Q, I)}(\forall \bar{u})(P(\bar{u}) \equiv Q(\bar{u}))$, where $I$ is a binary predicate constant not in $L, Q$ is a $k$-ary predicate constant not in $L, T(Q)$ is the set of sentences obtained from $T(P)$ by replacing all the occurrences of $P$ by $Q, \operatorname{Isom}_{L}(I ; P, Q)$ is the set of sentences expressing that $I$ is an isomorphism which is identity on the formulas in $L$ and such that it corresponds the predicate $P$ to $Q$. Notice that the condition (i)' is equivalent to (i) in the classical logic $L$. Therefore, by replacing (i) by (i)', we can consider two possible questions, Q1: whether or not (i)' is equivalent to (ii) in the case of $L=I L, \mathrm{Q} 2$ : whether or not (i)' is equivalent to (iii) in the case of $L=I L$. The answer for Q1 is negative (A counterexample will be given in $\S 4$ ). On the other hand, the answer for Q2, as it turns out, is affirmative. These are our assertions. Moreover it should be noted that we can know these facts only by a syntactical method.

This paper consists of four sections. After defining a Motohashi $P$ formula explicitly in $\S 1$, we shall state our Main theorem in $\S 2$, which will be proved in $\S 3$. Our entire proof in $\S 3$ will be carried out concretely by a proof-theoretic method.

\section{§1. Preliminaries}

Let $L$ be a first order language with equality symbol $=, P$ and $Q$ two fixed $k$-ary predicate constant symbols not in $L$, and $I$ a fixed binary predicate constant symbol not in $L$. We distinguish free variables (denoted by $x, y, \cdots$ with or without subscripts) from bound variables (denoted by $u, v, \cdots$ with or without subscripts) in $L$. Assume that the free variables in $L$ are divided into two infinite disjoint sets $X$ and $Y$. We denote by $I L$, the intuitionistic predicate logic with equality and by $\mathscr{L}$, the logic $I L(P, Q, I)$ obtained from $I L$ by adding all of $P, Q$ and $I$.

The class $\mathscr{M}(P)$ of Motohashi P-formulas in $L(P)$ is the smallest class satisfying the following three conditions:

(1) Each atomic formula in $L$ whose free variables are among $X$ is in $\mathscr{M}(P)$.

(2) Each atomic formula in $L(P)$ whose free variables are among $Y$ is in $\mathscr{M}(P)$.

(3) $\mathscr{M}(P)$ is closed under $\neg, \wedge, \vee, \supset, \forall$ and $\exists$.

It should be noted that $P\left(y_{1}, \cdots, y_{k}\right)$ is in $\mathscr{M}(P)$ for any $y_{1}, \cdots, y_{k}$ in $Y$ but $P\left(x_{1}, \cdots, x_{k}\right)$ is not in $\mathscr{M}(P)$ for any $x_{1}, \cdots, x_{k}$ in $X$. 


\section{§2. Main Theorem}

Let $T(P)$ be a set of sentences in $L(P)$ and $T(Q)$ the corresponding set of sentences of $L(Q)$ formed by replacing the occurrences of $P$ everywhere by $Q$. Suppose $\operatorname{Isom}_{L}(I ; P, Q)$ is the set of sentences:

$$
\begin{aligned}
& (\forall u)(\exists v) I(u, v), \quad(\forall v)(\exists u) I(u, v) \\
& (\forall \bar{u})(\forall \bar{v})(I(\bar{u}, \bar{v}) \cdot \supset \cdot P(\bar{u}) \equiv Q(\bar{v})), \\
& (\forall \bar{u})(\forall \bar{v})(I(\bar{u}, \bar{v}) . \supset \cdot \eta(\bar{u}) \equiv \eta(\bar{v})),
\end{aligned}
$$

where $\eta(\bar{x})$ is an atomic formula in $L$; the sequences $\bar{u}$ and $\bar{v}$ of bound variables have the same length and if $\bar{u}=\left\langle u_{1}, \cdots, u_{n}\right\rangle, \bar{v}=\left\langle v_{1}, \cdots, v_{n}\right\rangle$, then $I(\bar{u}, \bar{v})$ is an abbreviation for $I\left(u_{1}, v_{1}\right) \wedge \cdots \wedge I\left(u_{n}, v_{n}\right)$. Then our Main theorem states:

MaIN Theorem (Definability theorem for the intuitionistic predicate logic). The following two conditions are equivalent:

(1) $\operatorname{Isom}_{L}(I ; P, Q), T(P), T(Q) \vdash_{\mathscr{L}}(\forall \bar{u})(P(\bar{u}) \equiv Q(\bar{u}))$.

(2) $T(P) \vdash_{I L(P)}(\forall \bar{u})(P(\bar{u}) \equiv \varphi(\bar{u}))$ for some Motohashi P-formula $\varphi(\bar{x})$ in $L(P)$ whose free variables are among $\bar{x} \subseteq X$.

Remark. Unfortunately, the condition (2) does not mean that $T(P)$ defines $P$ explicitly, because $\varphi$ may have the predicate symbol $P$. But, we can not take $P(\bar{x})$ itself as $\varphi(\bar{x})$, since $P(\bar{x})$ is not a Motohashi $P$-formula in $L(P)$. I am sure that our Main theorem is the most general form of Svenonius-type definability theorem that we can hope for in the case of the intuitionistic predicate logic. The reason for this contention will be discussed in $\S 4$.

\section{§3. A proof of Main theorem}

Throughout this section, we assume that the language $L$ has no individual constant symbol or function constant symbol but that it has two propositional constant symbols $T, \perp$ for simplicity and that the logic $I L$ (hence $I L(P), \mathscr{L}$ ) is formulated in Gentzen style whose axiom sequents are as follows:

(1) $\longrightarrow$ T.

(2) $\perp \longrightarrow$.

(3) $\longrightarrow x=x$, where $x$ is a free variable.

(4) $E\left(x_{1}, y_{1}\right), \cdots, E\left(x_{m}, y_{m}\right), R\left(x_{1}, \cdots, x_{m}\right) \longrightarrow R\left(y_{1}, \cdots, y_{m}\right)$,

where $R$ is an $m$-ary predicate constant symbol, $x_{1}, \cdots, x_{m}$ and $y_{1}, \cdots, y_{m}$ 
are free variables, and $E\left(x_{i}, y_{i}\right)$ is an equality chain for $x_{i}$ to $y_{i}$ for each $1 \leqslant i \leqslant m$, that is, $E\left(x_{i}, y_{i}\right)$ is a set of the form $\left\{E_{1}, \cdots, E_{n}\right\}$ such that for each $1 \leqslant j \leqslant n, E_{j}$ is a formula either of the form $z_{j}=z_{j+1}$ or $z_{j+1}=z_{j}$, and $z_{1}=x_{i}, z_{n+1}=y_{i}$, where $z_{1}, \cdots, z_{n+1}$ are free variables.

An axiom sequent of the form (4) is called an axiom sequent for the predicate $R$, and if all $E\left(x_{i}, y_{i}\right)$ are empty sets in (4) then it (i.e., an axiom sequent of the form $\left.R\left(x_{1}, \cdots, x_{m}\right) \longrightarrow R\left(x_{1}, \cdots, x_{m}\right)\right)$ is said to be an identity axiom sequent for $R$, otherwise, it is said to be an equality axiom sequent for $R$. We use the usual inference rules (for example, rules in $L J$ ). By I-quantification rules, we mean the quantification rules whose principal formulas have the predicate $I$. For any language $L n$, a quantification rule whose principal formula is in $L n$ is called an Ln-quantification rule.

Lemma 1. Suppose that $\varphi(P ; \bar{y})$ is a Motohashi $P$-formula in $L(P)$ and $\bar{y}$ is a finite sequence of distinct free variables including all members of $Y$ occurring in $\varphi$. If a sequence $\bar{y}^{\prime}$, whose length is the same as that of $\bar{y}$, consists of distinct free variables not occurring in $\varphi$, then

$$
\operatorname{Isom}_{L}(I ; P, Q) \vdash_{\mathscr{L}} I\left(\bar{y}, \bar{y}^{\prime}\right) \longrightarrow \varphi(P ; \bar{y}) \equiv \varphi\left(Q ; \bar{y}^{\prime}\right) .
$$

Lemma 1 is easily proved by induction on the complexity of $\varphi(P ; \bar{y})$ from the definition $\operatorname{Isom}_{L}(I ; P, Q)$, and if we use this lemma, it is obviously shown that the condition (2) implies the condition (1) in our Main theorem.

Now we proceed to prove that (1) implies (2). For this it is sufficient to show that the following theorem holds.

ThEOREM. Suppose that $\bar{x}$ is a finite sequence of distinct free variables and $\theta(P ; \bar{x}), \psi(Q ; \bar{x})$ are formulas in $L(P), L(Q)$, respectively, all of whose free variables are chosen from $\bar{x}$. If

$$
\operatorname{Isom}_{L}(I ; P, Q) \vdash_{\mathscr{S}} \theta(P ; \bar{x}) \longrightarrow \psi(Q ; \bar{x}),
$$

then there exists a Motohashi P-formula $\varphi(\bar{x})$ in $L(P)$ whose free variables are among $\bar{x}$ such that the two sequents

$$
\theta(P ; \bar{x}) \longrightarrow \varphi(\bar{x}) \text { and } \varphi(\bar{x}) \longrightarrow \psi(P ; \bar{x})
$$

are both provable in $I L(P)$.

Proof of theorem. We assume that

$$
\operatorname{Isom}_{L}(I ; P, Q) \vdash_{\mathscr{L}} \theta(P ; \bar{x}) \longrightarrow \psi(Q ; \bar{x}) .
$$


Then, for some finite subset $\mathscr{I}$ of $\operatorname{Isom}_{L}(I ; P, Q)$, we get a derivation in $\mathscr{L}\left(\right.$ say, $\left.\mathscr{D}_{1}\right)$ which has the sequent

$$
\mathscr{I}, \theta(P ; \bar{x}) \longrightarrow \psi(Q ; \bar{x})
$$

as its end-sequent. The sequential transformations Step 1 Step 5 mentioned below give us a tree form $\mathscr{D}_{6}$ that is enough for us to prove the theorem in a simpler manner.

Step 1: Elimination of the equality axiom sequents for the predicate $I$.

Step 2: Elimination of the cut rules.

Step 3: Specialization of the I-quantification rules.

Step 4: Elimination of the identity axiom sequents for the predicate $I$.

Step 5: Elimination of the left weakening rules with respect to the predicate $I$.

We shall denote by $\mathscr{D}_{i+1}$, the new tree form obtained from $\mathscr{D}_{i}$ by carrying out the transformation of Step $i$ for each $1 \leqslant i \leqslant 5$. Since a new tree form $\mathscr{D}_{i}(i=4,5,6)$ may contain topmost sequents which are not axiom sequents in $\mathscr{L}$ or inference rules which do not belong to $\mathscr{L}$, it is not a derivation in $\mathscr{L}$. But, for simplicity, it is also called a derivation in $\mathscr{L}$ as long as there is no confusion in proving the theorem.

We let $I_{\sharp}(x, y)$ be an abbreviation for $(\exists u)(\exists v)(x=u \wedge y=v \wedge I(u, v))$, and if $\bar{x}, \bar{y}$ are sequences of free variables of the same length $n$, and $\bar{u}, \bar{v}$ are sequences of bound variables of the same length $n$, then $I_{\sharp}(\bar{x}, \bar{y})$ means $(\exists \bar{u})(\exists \bar{v})(\bar{x}=\bar{u} \wedge \bar{y}=\bar{v} \wedge I(\bar{u}, \bar{v}))$ i.e., $\left(\exists u_{1}\right) \cdots\left(\exists u_{n}\right)\left(\exists v_{1}\right) \cdots\left(\exists v_{n}\right)\left(x_{1}=u_{1} \wedge \cdots\right.$ $\left.\wedge x_{n}=u_{n} \wedge y_{1}=v_{1} \wedge \cdots \wedge y_{n}=v_{n} \wedge I\left(u_{1}, v_{1}\right) \wedge \cdots \wedge I\left(u_{n}, v_{n}\right)\right)$.

Step 1. Elimination of the equality axiom sequents for the predicate $I$.

LEMma 2.1. The sequent

$$
E\left(x_{1}, y_{1}\right), E\left(x_{2}, y_{2}\right), I_{\sharp}\left(x_{1}, x_{2}\right) \longrightarrow I_{\sharp}\left(y_{1}, y_{2}\right)
$$

is provable in $\mathscr{L}$ without equality axiom sequents for $I$, where $E\left(x_{i}, y_{i}\right)$ is an equality chain for $x_{i}$ to $y_{i}(i=1,2)$.

LemMa 2.2. The following four sequents are provable in $\mathscr{L}$ without equality axiom sequents for $I$ :

(i) $(\forall u)(\exists v) I(u, v) \longrightarrow(\forall u)(\exists v) I_{\sharp}(u, v)$,

(ii) $(\forall v)(\exists u) I(u, v) \longrightarrow(\forall v)(\exists u) I_{\sharp}(u, v)$, 
(iii) $(\forall \bar{u})(\forall \bar{v})\left(\forall \bar{u}^{\prime}\right)\left(\forall \bar{v}^{\prime}\right)\left(\bar{u}=\bar{u}^{\prime} \wedge \bar{v}=\bar{v}^{\prime} \wedge I\left(\bar{u}^{\prime}, \bar{v}^{\prime}\right) \wedge P(\bar{u}) . \supset . Q(\bar{v})\right) \wedge$ $(\forall \bar{u})(\forall \bar{v})\left(\forall \bar{u}^{\prime}\right)\left(\forall \bar{v}^{\prime}\right)\left(\bar{u}=\bar{u}^{\prime} \wedge \bar{v}=\bar{v}^{\prime} \wedge I\left(\bar{u}^{\prime}, \bar{v}^{\prime}\right) \wedge Q(\bar{v}) . \supset . P(\bar{u})\right)$ $\longrightarrow(\forall \bar{u})(\forall \bar{v})\left(I_{\#}(\bar{u}, \bar{v}) . \supset . P(\bar{u}) \equiv Q(\bar{v})\right)$,

(iv) $(\forall \bar{u})(\forall \bar{v})\left(\forall \bar{u}^{\prime}\right)\left(\forall \bar{v}^{\prime}\right)\left(\bar{u}=\bar{u}^{\prime} \wedge \bar{v}=\bar{v}^{\prime} \wedge I\left(\bar{u}^{\prime}, \bar{v}^{\prime}\right) \wedge \eta(\bar{u}) . \supset . \eta(\bar{v})\right) \wedge$ $(\forall \bar{u})(\forall \bar{v})\left(\forall \bar{u}^{\prime}\right)\left(\forall \bar{v}^{\prime}\right)\left(\bar{u}=\bar{u}^{\prime} \wedge \bar{v}=\bar{v}^{\prime} \wedge I\left(\bar{u}^{\prime}, \bar{v}^{\prime}\right) \wedge \eta(\bar{v}) . \supset . \eta(\bar{u})\right)$ $\longrightarrow(\forall \bar{u})(\forall \bar{v})\left(I_{\sharp}(\bar{u}, \bar{v}) . \supset . \eta(\bar{u}) \equiv \eta(\bar{v})\right)$,

where $\eta(\bar{x})$ is an atomic formula in $L$.

Now, for each set $\Gamma$ of formulas in $\mathscr{L}$ we denote by $\Gamma^{\sharp}$, the set of formulas which results from $\Gamma$ by replacing the parts of the form $I(x, y)$ in each formula in $\Gamma$ by $I_{\#}(x, y)$ simultaneously.

Let $\mathscr{D}_{1}^{\sharp}$ be the tree form obtained from $\mathscr{D}_{1}$ by substituting $\Gamma^{\sharp} \rightarrow \Theta^{\sharp}$ for each sequent $\Gamma \rightarrow \Theta$ which occurs in $\mathscr{D}_{1}$. Let $\tilde{\mathscr{D}}_{1}$ be the tree form obtained from $\mathscr{D}_{1}^{\sharp}$ by adding, over the sequent $\Pi^{\sharp} \rightarrow \Lambda^{\sharp}$, the derivation $\mathscr{E}$ with endsequent $\Pi^{\sharp} \rightarrow \Lambda^{\sharp}$, where the sequent $\Pi^{\sharp} \rightarrow \Lambda^{\sharp}$ ranges over all of the topmost sequents in $\mathscr{D}_{1}^{*}$, each of which is corresponding to an axiom sequent for the predicate $I$, say $\Pi \rightarrow \Lambda$; and the existence of the derivation $\mathscr{E}$ is guaranteed by Lemma 2.1. This $\widetilde{\mathscr{D}}_{1}$ is a derivation with end-sequent $\mathscr{I}^{\sharp}$, $\theta(P ; \bar{x}) \rightarrow \psi(Q ; \bar{x})$ in $\mathscr{L}$. If we put $\mathscr{D}_{2}$ as follows, it becomes a derivation in $\mathscr{L}$ with end-sequent $\mathscr{I}^{*},(P ; \bar{x}) \rightarrow \psi(Q ; \bar{x})$ and without equality axiom sequents for $I$. $\mathscr{D}_{2}$ :

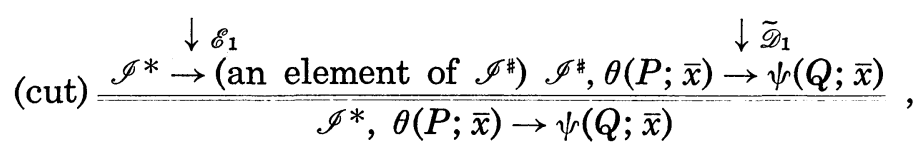

where $\mathscr{I}^{*}$ is the set obtained from $\mathscr{I}^{\#}$ by substituting: $(\forall u)(\exists v) I_{\sharp}(u, v)$ by $(\forall u)(\exists v) I(u, v) ;(\forall v)(\exists u) I_{\sharp}(u, v)$ by $(\forall v)(\exists u) I(u, v) ;(\forall \bar{u})(\forall \bar{v})\left(I_{\sharp}(\bar{u}, \bar{v}) . \supset . F(\bar{u})\right.$ $\equiv G(\bar{v}))$ by $(\forall \bar{u})(\forall \bar{v})\left(\forall \bar{u}^{\prime}\right)\left(\forall \bar{v}^{\prime}\right)\left(\bar{u}=\bar{u}^{\prime} \wedge \bar{v}=\bar{v}^{\prime} \wedge\left(I\left(\bar{u}^{\prime}, \bar{v}^{\prime}\right) \wedge F(\bar{u}) . \supset . G(\bar{v})\right)\right.$ $\wedge(\forall \bar{u})(\forall \bar{v})\left(\forall \bar{u}^{\prime}\right)\left(\forall \bar{v}^{\prime}\right)\left(\bar{u}=\bar{u}^{\prime} \wedge \bar{v}=\bar{v}^{\prime} \wedge I\left(\bar{u}^{\prime}, \bar{v}^{\prime}\right) \wedge G(\bar{v}) . \supset . F(\bar{u})\right)$, where $(F, G)$ is either the pair $(P, Q)$ of the predicates or the pair $(\eta, \eta)$ of an atomic formula $\eta$ in $L$. (This sentence is said to be of type $(F, G)$.) The existence of $\mathscr{E}_{1}$ is guaranteed by Lemma 2.2. Double lines $=$ mean that

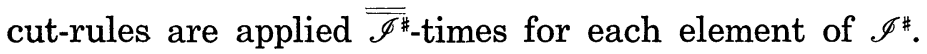

Step 2. Elimination of the cut rules.

This transformation is done in the usual way for the derivation $\mathscr{D}_{2}$, and the new derivation $\mathscr{D}_{3}$ satisfies the following conditions:

(a) Its end-sequent is $\mathscr{I}^{*}, \theta(P ; \bar{x}) \longrightarrow \psi(Q ; \bar{x})$, 
(b) It satisfies the eigenvariable conditions and it has no equality axiom sequent for $I$ nor cut-rule.

Step 3. Specialization of the I-quantification rules.

From the condition (b), $\mathscr{D}_{3}$ has the subformula property. Formulas which occur in each sequent in $\mathscr{D}_{3}$, therefore, are subformulas of the formulas occurring in the end-sequent, and so each formula including the predicate $I$ is a subformula of a sentence in $\mathscr{I}^{*}$.

Definitions. For each sentence $\varphi$ in $\mathscr{I}^{*}$, we define $(\varphi)$-rules as follows:

(1) $\varphi=(\forall u)(\exists v) I(u, v)$, then

$$
(\varphi) \frac{I(x, z), \Gamma \longrightarrow \Theta}{\Gamma \longrightarrow \Theta}
$$

where $z$ is different from $x$ and it does not occur in the lower sequent.

(2) $\varphi=(\forall v)(\exists u) I(u, v)$, then

$$
(\varphi) \frac{I(z, x), \Gamma \longrightarrow \Theta}{\Gamma \longrightarrow \Theta}
$$

where $z$ is different from $x$ and it does not occur in the lower sequent.

(3) $\varphi$ is of type $(F, G)$, then the rule $(\varphi)$ is either $(\varphi)_{1}$ or $(\varphi)_{2}$ :

$$
\begin{gathered}
(\varphi)_{1} \frac{\Gamma \rightarrow \bar{x}=\bar{x}^{\prime} \Gamma \rightarrow \bar{y}=\bar{y}^{\prime} \Gamma \rightarrow I\left(x_{1}^{\prime}, y_{1}^{\prime}\right) \cdots \Gamma \rightarrow I\left(x_{n}^{\prime}, y_{n}^{\prime}\right) \Gamma \rightarrow F(\bar{x}) \Gamma, G(\bar{y}) \rightarrow \Theta}{\Gamma \longrightarrow \Theta} \\
(\varphi)_{2} \frac{\Gamma \rightarrow \bar{x}=\bar{x}^{\prime} \Gamma \rightarrow \bar{y}=\bar{y}^{\prime} \Gamma \rightarrow I\left(x_{1}^{\prime}, y_{1}^{\prime}\right) \cdots \Gamma \rightarrow I\left(x_{n}^{\prime}, y_{n}^{\prime}\right) \Gamma \rightarrow G(\bar{y}) \Gamma, F(\bar{x}) \rightarrow \Theta}{\Gamma \longrightarrow \Theta}
\end{gathered}
$$

where $\bar{x}, \bar{y}, \bar{x}^{\prime}, \bar{y}^{\prime}$ are sequences of free variables with the same length $n$ and $\bar{x}^{\prime}=\left\langle x_{1}^{\prime}, \cdots, x_{n}^{\prime}\right\rangle, \bar{y}^{\prime}=\left\langle y_{1}^{\prime}, \cdots, y_{n}^{\prime}\right\rangle$.

Let $S_{1}\left(\mathscr{I}^{*}\right)$ be the union of $\mathscr{I}^{*}$ and the set of all subformulas of a sentence in $\mathscr{I}^{*}$ whose outermost logical symbols are one of $\supset, \forall, \exists$. Let $S_{2}\left(\mathscr{I}^{*}\right)$ be the set of all subformulas of a sentence of type $(F, G)$ in $\mathscr{I}^{*}$ whose outermost logical symbol is $\wedge$.

Lemma 3. Suppose that $\mathscr{C}$ is a subderivation of $\mathscr{D}_{3}$ with end-sequent $\Gamma \rightarrow \Theta$, and $\Gamma^{*}$ is $\Gamma-S_{1}\left(\mathscr{I}^{*}\right)$.

(i) If $\Theta$ contains no element of $S_{2}\left(\mathscr{I}^{*}\right)$, then there is a der vation $\mathscr{C}^{*}$ such that (1) its end-sequent is $\Gamma^{*} \rightarrow \Theta$, (2) it contains no I-quantification rule, but it generally contains $(\varphi)$-rules for each sentence $\varphi$ in $\mathscr{I}^{*}$.

(ii) Otherwise, say $\Theta=\left\{F_{1} \wedge \cdots \wedge F_{n}\right\}$, then there are $n$-derivations $\mathscr{C}_{1}$, 
$\cdots, \mathscr{C}_{n}$ such that (1) their end-sequents are $\Gamma^{*} \rightarrow F_{1}, \cdots, \Gamma^{*} \rightarrow F_{n}$, respectively, and (2) they contain no I-quantification rule, but they generally contain $(\varphi)$-rules for each sentence $\varphi$ in $\mathscr{I}^{*}$.

Proof of Lemma 3. We prove the Lemma by induction on the complexity of the subderivation $\mathscr{C}$ of $\mathscr{D}_{3}$. If $\mathscr{C}$ is an axiom sequent then we have nothing to prove, so we assume that $\mathscr{C}$ is not an axiom sequent. In this case we divide the proof into several cases according to the last rule $R$ of $\mathscr{C}$. Here we take up only two cases, the proofs of all other cases being similar to one or the other.

Case 1. $R=(\supset \rightarrow)$. In this case $\mathscr{C}$ is of the form:

$$
R \frac{\Gamma \stackrel{\downarrow \mathscr{E}}{\longrightarrow} F_{1} \Gamma, F_{2} \stackrel{\downarrow \mathscr{F}}{\longrightarrow} \Theta}{F_{1} \supset F_{2}, \Gamma \longrightarrow \Theta}
$$

1.1 Suppose that $\Theta$ contains no element of $S_{2}\left(\mathscr{I}^{*}\right)$.

1.11. $F_{1} \supset F_{2} \in S_{1}\left(\mathscr{I}^{*}\right)$. Then $\left(F_{1} \supset F_{2}, \Gamma\right)^{*}=\Gamma^{*}$, and we may assume that $F_{1} \supset F_{2}$ is of the form $\bar{x}=\bar{x}^{\prime} \wedge \bar{y}=\bar{y}^{\prime} \wedge I\left(\bar{x}^{\prime}, \bar{y}^{\prime}\right) \wedge F(\bar{x}) . \supset . G(\bar{y})$, without loss of generality. By the induction hypothesis on $\mathscr{E}$ and $\Gamma \rightarrow F_{1}$ (resp. $\mathscr{F}$ and $\Gamma, F_{2} \rightarrow \Theta$ ), there are derivations $\mathscr{E}_{1}, \cdots, \mathscr{E}_{n+3}(n=$ length of $\bar{x}$ ) (resp. $\mathscr{F}^{*}$ ) which satisfy the conditions (1) and (2) on $\left\{F_{1}\right\}$ (resp. $\Theta$ ) of the Lemma. Then we put $\mathscr{C}^{*}$ as follows:

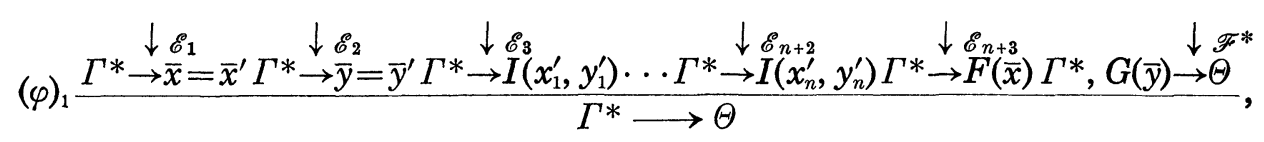

where $\varphi$ is of type $(F, G)$. This derivation $\mathscr{C}^{*}$ is the one we wanted.

1.12. $F_{1} \supset F_{2} \notin S_{1}\left(\mathscr{I}^{*}\right)$. In this case, $F_{2} \notin S_{1}\left(\mathscr{I}^{*}\right)$ and $\left(F_{1} \supset F_{2}, \Gamma\right)^{*}=$ $F_{1} \supset F_{2}, \Gamma^{*}$. By the induction hypothesis on $\mathscr{E}$ and $\Gamma \rightarrow F_{1}$ (resp. $\mathscr{F}$ and $\Gamma, F_{2} \rightarrow \Theta$ ), there is a derivation $\mathscr{E}^{*}$ (resp. $\mathscr{F}^{*}$ ) such that the conditions (1) and (2) hold. Hence we put $\mathscr{C}^{*}$ as:

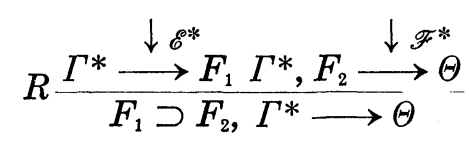

1.2. Not 1.1. Similar to case 1.1.

Case 2. $R=(\exists \rightarrow)$. $\mathscr{C}$ is of the form: 


$$
R \frac{F(z), \Gamma \stackrel{\downarrow \mathscr{E}}{\longrightarrow} \Theta}{(\exists v) F(v), \Gamma \longrightarrow \Theta},
$$

where $z$ does not occur in the lower sequent.

2.1. Suppose that $\Theta$ contains no element of $S_{2}\left(\mathscr{I}^{*}\right)$.

2.11. $(\exists v) F(v) \in S_{1}\left(\mathscr{I}^{*}\right)$. Then $((\exists v) F(v), \Gamma)^{*}=\Gamma^{*}$ and $(\exists v) F(v)$ must be either of the form $(\exists v) I(x, v)$ or of the form $(\exists v) I(v, x)$. We may assume that $(\exists v) F(v)=(\exists v) I(x, v)$. Hence $F(z)=I(x, z) \notin S_{1}\left(\mathscr{I}^{*}\right)$. From the induction hypothesis on $\mathscr{E}$ and $F(z), \Gamma \rightarrow \Theta$, we get a derivation $\mathscr{E} *$ which satisfies the conditions (1) and (2). Take $\mathscr{C}^{*}$ as

$$
(\varphi) \frac{I(x, z), \Gamma^{*} \stackrel{\downarrow \mathscr{E}^{*}}{\longrightarrow} \Theta}{\Gamma^{*} \longrightarrow \Theta},
$$

where $\varphi=(\forall u)(\exists v) I(u, v)$.

2.12. $(\exists v) F(v) \notin S_{1}\left(\mathscr{I}^{*}\right)$. Then $F(z) \notin S_{1}\left(\mathscr{I}^{*}\right),((\exists v) F(v), \Gamma)^{*}=(\exists v) F(v)$, $\Gamma^{*}$, and $F(z)$ must be a formula in $L(P, Q)$. Hence $R$ is an $L(P, Q)$-quantification rule. By the induction hypothesis on $\mathscr{E}$ and $F(z), \Gamma \rightarrow \Theta$, we get a derivation $\mathscr{E}^{*}$ which satisfies the conditions (1) and (2). It is enough to take $\mathscr{C}^{*}$ as

$$
R \frac{F(z), \Gamma^{*} \stackrel{\downarrow \mathscr{E}^{*}}{\longrightarrow} \Theta}{(\exists v) F(v), \Gamma^{*} \longrightarrow \Theta} .
$$

\subsection{Not 2.1. Similar to case 2.1.}

Now we apply Lemma 3 for the derivation $\mathscr{D}_{3}$ and its end-sequent $\mathscr{I}^{*}, \theta(P ; \bar{x}) \rightarrow \psi(Q ; \bar{x})$, and we get a derivation $\mathscr{D}_{3}^{*}$ (say, $\left.\mathscr{D}_{4}\right)$ such that:

(a) Its end-sequent is $\theta(P ; \bar{x}) \rightarrow \psi(Q ; \bar{x})$,

(c) No $I$-quantification rule occurs in it and it contains $(\varphi)$-rules for each sentence $\varphi$ in $\mathscr{I}^{*}$.

Moreover, it is obvious that $\mathscr{D}_{4}$ satisfies the condition (b) and the following condition:

(d) It has the I-eigenvariable property, that is, at an instance of some rule in $\mathscr{D}_{4}$, an atomic formula of the form $I(x, y)$ occurring in the antecedent of the upper sequent but not occurring in the antecedent of the lowe: sequent has the eigenvariables of the rule. 
Step 4. Elimination of the identity axiom sequents for the predicate $I$.

LEMma 4. Suppose that $\mathscr{D}$ is a subderivation of $\mathscr{D}_{4}$ with end-sequent $\Gamma \rightarrow \Theta$, and $I(x, y)$ does not occur in $\Gamma$. Then there exists a derivation $\mathscr{D}^{*}$ such that (1) its end-sequent is $\Gamma \rightarrow \Theta^{*}$, where $\Theta^{*}=\Theta-\{I(x, y)\}$ (hence it is an empty set or $\Theta$ itself), (2) it contains no I-quantification rule, and it contains $(\varphi)$-rules for $\varphi=(\forall u)(\exists v) I(u, v)$ or $(\forall v)(\exists u) I(u, v)$ and $(\varphi)^{*}$-rules for each sentence $\varphi$ of type $(F, G)$ in $\mathscr{I}^{*}$, where $a(\varphi)^{*}$-rule is either a $(\varphi)_{1}^{*}$ rule or $a(\varphi)_{2}^{*}$-rule as follows:

$$
\begin{gathered}
(\varphi)_{1}^{*} \frac{\Gamma \longrightarrow \bar{x}=\bar{x}^{\prime} \Gamma \longrightarrow \bar{y}=\bar{y}^{\prime} \Gamma \longrightarrow F(\bar{x}) \Gamma, G(\bar{y}) \longrightarrow \Theta}{\Gamma \longrightarrow \Theta} \\
(\varphi)_{2}^{*} \stackrel{\Gamma \longrightarrow \bar{x}=\bar{x}^{\prime} \Gamma \longrightarrow \bar{y}=\bar{y}^{\prime} \Gamma \longrightarrow G(\bar{y}) \Gamma, F(\bar{x}) \longrightarrow \Theta}{\Gamma \longrightarrow \Theta},
\end{gathered}
$$

where $\Gamma$ contains the set $\left\{I\left(\bar{x}^{\prime}, \bar{y}^{\prime}\right)\right\}=\left\{I\left(x_{1}^{\prime}, y_{1}^{\prime}\right), \cdots, I\left(x_{n}^{\prime}, y_{n}^{\prime}\right)\right\}$ as a subset and $\bar{x}, \bar{x}^{\prime}, \bar{y}, \bar{y}^{\prime}$ are sequences of free variables with the same length.

Let $\mathscr{D}_{5}$ be the derivation obtained from $\mathscr{D}_{4}$ by applying the above Lemma 4 to $\mathscr{D}_{4}$ and its end-sequent $\theta(P ; \bar{x}) \rightarrow \psi(Q ; \bar{x})$. Then $\mathscr{D}_{5}$ clearly satisfies the conditions (a)' and (b). Moreover, none of the identity axiom sequents for $I$ are contained in $\mathscr{D}_{5}$ because $\mathscr{D}_{4}$ has the $I$-eigenvariable property.

Step 5. Elimination of the left weakening rules with respect to $I$.

Any occurrence of an atomic formula of the form $I(x, y)$ in the antecedent of each sequent in $\mathscr{D}_{5}$ is due to left weakening rules with respect to $I$ because $\mathscr{D}_{5}$ has no axiom sequent for $I$. We then remove all of the atomic formulas of the form $I(x, y)$ occurring in the antecedent of each sequent in $\mathscr{D}_{5}$ and we add them to the antecedent of each topmost sequent in $\mathscr{D}_{5}$. In this manner we obtain a new tree form $\mathscr{D}_{6}$ from $\mathscr{D}_{5}$. If we admit, as an axiom sequent in $\mathscr{L}$, a new sequent obtained from an axiom sequent by adding atomic formulas of the form $I(x, y)$ in the antecedent of it and also admit $(\varphi)$-rules for $\varphi=(\forall u)(\exists v) I(u, v)$ or $(\forall v)(\exists u)$ $I(u, v)$ and $(\varphi)^{*}$-rules for a sentence $\varphi$ of type $(F, G)$ in $\mathscr{I}^{*}$ as an inference rule in $\mathscr{L}$, then $\mathscr{D}_{6}$ becomes our desired derivation in $\mathscr{L}$.

Therefore each sequent occurring in $\mathscr{D}_{6}$ is of the form:

$$
\{I(\bar{x}, \bar{y})\}, \Gamma_{1}(P), \Gamma_{2}(Q) \longrightarrow \Theta_{1}(P), \Theta_{2}(Q),
$$

where $\bar{x}$ and $\bar{y}$ are sequences of free variables with the same length and if $\bar{x}=\left\langle x_{1}, \cdots, x_{n}\right\rangle, \bar{y}=\left\langle y_{1}, \cdots, y_{n}\right\rangle$, then $\{I(\bar{x}, \bar{y})\}$ means $\left\{I\left(x_{1}, y_{1}\right), \cdots, I\left(x_{n}, y_{n}\right)\right\}$. 
Besides, $\Gamma_{1}(P), \Theta_{1}(P)$ are sets of formulas in $L(P) ; \Gamma_{2}(Q), \Theta_{2}(Q)$ are sets of formulas in $L(Q)$, and $\Theta_{1}(P) \cup \Theta_{2}(Q)$ has at most one element.

Definitions. A 6-tuple $\Delta=\left\langle\bar{x}, \bar{y} ; \Gamma_{1}(P), \Gamma_{2}(Q) ; \Theta_{1}(P), \Theta_{2}(Q)\right\rangle$ is called an I-partition of a sequent $\Gamma \rightarrow \Theta$ if it satisfies the conditions (1)-(3):

(1) $\Gamma-\{I(\bar{x}, \bar{y})\}=\Gamma_{1}(P), \Gamma_{2}(Q)$ and $\Theta=\Theta_{1}(P), \Theta_{2}(Q)$.

(2) $\bar{x}$ (resp. $\bar{y}$ ) is a finite sequence of free variables which includes all the free variables occurring in $\Gamma_{1}(P) \cup \Theta_{1}(P)$ (resp. $\Gamma_{2}(Q) \cup \Theta_{2}(Q)$ ). Furthermore, if a free variable $x_{i}$ of $\bar{x}$ occurs both in $\Gamma_{1}(P) \cup \Theta_{1}(P)$ and in $\Gamma_{2}(Q) \cup \Theta_{2}(Q)$, then so is $y_{i}$ of $\bar{y}$ (with the same index), and vice versa.

( 3 ) $\Gamma_{1}(P), \Theta_{1}(P)$ (resp. $\Gamma_{2}(Q), \Theta_{2}(Q)$ ) are sets of formulas in $L(P)$ (resp. $L(Q)$ ), and $\Theta_{1}(P) \cup \Theta_{2}(Q)$ consists of at most one element.

A sequent is said to be I-partitionable if it has an I-partition. It is obvious that each sequent in $\mathscr{D}_{6}$ is $I$-partitionable. This is due to the fact that the end-sequent $\theta(P ; \bar{x}) \rightarrow \psi(Q ; \bar{x})$ in $\mathscr{D}_{6}$ has the $I$-partition $\Delta=\langle\bar{x}, \bar{x}$; $\{\theta(P ; \bar{x})\}, \emptyset ; \emptyset,\{\psi(Q ; \bar{x})\}\rangle$ and at any instance of an inference rule in $\mathscr{D}_{6}$, if the lower sequent is $I$-partitionable then the upper sequent is $I$-partitionable.

It now remains for us to prove the following proposition. Let $F V$ be the set of all free variables in $L$ and $F V\left(\mathscr{D}_{6}\right)$ be the set of all free variables occurring in $\mathscr{D}_{6}$. Fix a one-one function $f$ from $F V^{2}$ into $X$ such that range $(f)$ is infinite and range $(f) \subseteq X-F V\left(\mathscr{D}_{6}\right)$.

Proposition. Suppose that $\mathscr{D}$ is any subderivation of $\mathscr{D}_{6}$, and $\Delta=$ $\left\langle\bar{x}, \bar{y} ; \Gamma_{1}(P), \Gamma_{2}(Q) ; \Theta_{1}(P), \Theta_{2}(Q)\right\rangle$ is any I-partition of the end-sequent of $\mathscr{D}$. If $\Theta_{1}(P)$ is empty (resp. $\Theta_{2}(Q)$ is empty), then there exists a formula $\varphi$ in $\mathscr{M}(P)$ such that:

(1) each free variable in $\varphi$ appears in $f(\bar{x}, \bar{y})$,

(2) the two sequents

$$
\begin{gathered}
\Gamma_{1}(P) \longrightarrow \varphi\left(\begin{array}{c}
f(\bar{x}, \bar{y}) \\
\bar{x}
\end{array}\right) \text { and } \varphi\left(\begin{array}{c}
f(\bar{x}, \bar{y}) \\
\bar{y}
\end{array}\right), \Gamma_{2}(P) \longrightarrow \Theta_{2}(P) \\
\left(\operatorname{resp.} \varphi\left(\begin{array}{c}
f(\bar{x}, \bar{y}) \\
\bar{x}
\end{array}\right), \Gamma_{1}(P) \longrightarrow \Theta_{1}(P) \text { and } \quad \Gamma_{2}(P) \longrightarrow \varphi\left(\begin{array}{c}
f(\bar{x}, \bar{y}) \\
\bar{y}
\end{array}\right)\right)
\end{gathered}
$$

are both provable in $I L(P)$, where, if $\bar{x}=\left\langle x_{1}, \cdots, x_{n}\right\rangle$ and $\bar{y}=\left\langle y_{1}, \cdots, y_{n}\right\rangle$, then $f(\bar{x}, \bar{y})=\left\langle f\left(x_{1}, y_{1}\right), \cdots, f\left(x_{n}, y_{n}\right)\right\rangle, \varphi\left(\frac{f(\bar{x}, \bar{y})}{\bar{x}}\right)$ denotes the formula obtained from $\varphi$ by replacing each $f\left(x_{i}, y_{i}\right)$ by $x_{i}(1 \leqslant i \leqslant n)$. Similarly with $\varphi\left(\begin{array}{c}f(\bar{x}, \bar{y}) \\ \bar{y}\end{array}\right)$. Moreover, $\Gamma_{2}(P)$ and $\Theta_{2}(P)$ are sets derived from $\Gamma_{2}(Q)$ and $\Theta_{2}(Q)$ respectively by replacing every occurrence of the predicate $Q$ in each formula 
of $\Gamma_{2}(Q)$ and $\Theta_{2}(Q)$ by the predicate $P$.

Proof of Proposition. The proof is by induction on the complexity of $\mathscr{D}$. When $\mathscr{D}$ is not an axiom sequent, we divide the proof into five cases according to the last rule $R$ of $\mathscr{D}$.

Case 1. $R$ is one of the rules $(w \rightarrow),(\rightarrow w),(\neg \rightarrow),(\rightarrow \neg),(\wedge \rightarrow),(\rightarrow \vee)$, $(\rightarrow \supset)$. In this case, our desired formula $\varphi$ may be the formula itself obtained via the induction hypothesis.

Case 2. $R$ is one of the rules $(\rightarrow \wedge),(\vee \rightarrow),(\supset \rightarrow)$. In this case, our desired formula $\varphi$ is the resulting from $\varphi_{1}, \varphi_{2}$ by connecting one of $\wedge, \vee$, $\supset$, where $\varphi_{1}, \varphi_{2}$ are formulas obtained from the induction hypothesis.

Case $3 . \quad R$ is an $L(P, Q)$-quantification rule. We only consider the case $R=(\forall \rightarrow)$. $\mathscr{D}$ is of the form:

$$
R \frac{F(x), \Gamma \stackrel{\downarrow \mathscr{E}}{\longrightarrow} \Theta}{\longrightarrow}
$$

Let $A=\left\langle\bar{x}, \bar{y} ; \Gamma_{1}(P), \Gamma_{2}(Q) ; \Theta_{1}(P), \Theta_{2}(Q)\right\rangle$ be an $I$-partition of the lower sequent $(\forall v) F(v), \Gamma \rightarrow \Theta$. Without loss of generality, we may assume that $F,(\forall v) F(v)$ are not in $\Gamma$.

Subcase 3.1. $(\forall v) F(v) \in \Gamma_{1}(P)$. Take $\Gamma_{1}(P)^{\prime}=\Gamma_{1}(P)-\{(\forall v) F(v)\}$.

a) $x \notin \bar{x} \cap \bar{y} . \quad \Delta^{\prime}=\left\langle\bar{x} \frown x, \bar{y} \frown x ; \Gamma_{1}(P)^{\prime} \cup\{F(x)\}, \Gamma_{2}(Q) ; \Theta_{1}(P), \Theta_{2}(Q)\right\rangle$ is an $I$-partition of the upper sequent $F(x), \Gamma \rightarrow \Theta$. From the induction hypothesis on $\mathscr{E}$ and $\Delta^{\prime}$, we get a formula $\varphi^{\prime}(f(\bar{x}, \bar{y}), f(x, x))$ in $\mathscr{M}(P)$ which satisfies (1), (2) on $\Delta^{\prime}$. If $\Theta_{1}(P)$ is empty (resp. $\Theta_{2}(Q)$ is empty), therefore, the two sequents

$$
\begin{aligned}
& \Gamma_{1}(P)^{\prime}, F(x) \rightarrow \varphi^{\prime}\left(\begin{array}{c}
f(\bar{x}, \bar{y}), f(x, x) \\
\bar{x}, \quad x
\end{array}\right) \text { and } \varphi^{\prime}\left(\begin{array}{c}
f(\bar{x}, \bar{y}), f(x, x) \\
\bar{y}, x
\end{array}\right), \Gamma_{2}(P) \rightarrow \Theta_{2}(P)
\end{aligned}
$$

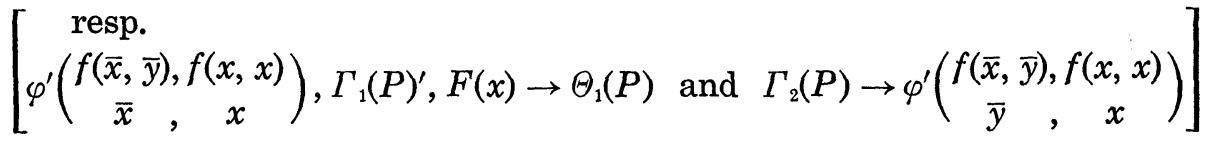

are provable in $I L(P)$. Hence it is enough to put $\varphi=(Q v) \varphi^{\prime}(f(\bar{x}, \bar{y}), v)$, where the quantifier $Q$ is defined as follows:

$$
Q= \begin{cases}\exists(\text { resp. } \forall) & \text { if } x \in \bar{x}-\bar{y} \\ \forall(\text { resp. } \exists) & \text { o.t. }\end{cases}
$$


b) $x \in \bar{x} \cap \bar{y}$. By the induction hypothesis on $\mathscr{E}$ and an $I$-partition $\Delta^{\prime}=\left\langle\bar{x}, \bar{y} ; \Gamma_{1}(P)^{\prime} \cup\{F(x)\}, \Gamma_{2}(Q) ; \Theta_{1}(P), \Theta_{2}(Q)\right\rangle$ of the upper sequent, we have a formula $\varphi$ in $\mathscr{M}(P)$ which satisfies conditions (1), (2) on $\Delta^{\prime}$ and also conditions (1), (2) on 4 .

Subcase 3.2. $(\forall v) F(v) \in \Gamma_{2}(Q)$. Similar to subcase 3.1.

Case 4. $R=(\psi)$ with $\psi=(\forall u)(\exists v) I(u, v)$ or $(\forall v)(\exists u) I(u, v)$. We may assume that $\psi=(\forall u)(\exists v) I(u, v)$. Then $\mathscr{D}$ is of the form:

$$
R \frac{I(x, z), \Gamma \stackrel{\downarrow \mathscr{E}}{\longrightarrow} \Theta}{\Gamma \longrightarrow \Theta}
$$

where $z$ is different from $x$ and it does not occur in $\Gamma \rightarrow \Theta$.

Let $\Delta=\left\langle\bar{x}, \bar{y} ; \Gamma_{1}(P), \Gamma_{2}(Q) ; \Theta_{1}(P), \Theta_{2}(Q)\right\rangle$ be an $I$-partition of the lower sequent $\Gamma \rightarrow \Theta$. Then $\Delta^{\prime}=\left\langle\bar{x} \frown x, \bar{y} \frown z ; \Gamma_{1}(P), \Gamma_{2}(Q) ; \Theta_{1}(P), \Theta_{2}(Q)\right\rangle$ is an $I-$ partition of the upper sequent $I(x, z), \Gamma \rightarrow \Theta$. From the induction hypothesis on $\mathscr{E}$ and $\Delta^{\prime}$, we get a formula $\varphi^{\prime}(f(\bar{x}, \bar{y}), f(x, z))$ in $\mathscr{M}(P)$ such that conditions (1), (2) on $\Delta^{\prime}$ hold. By the condition (2) on $\Delta^{\prime}$, if $\Theta_{1}(P)$ is empty (resp. $\Theta_{2}(Q)$ is empty), then two sequents

$$
\begin{gathered}
\Gamma_{1}(P) \rightarrow \varphi^{\prime}\left(\begin{array}{c}
f(\bar{x}, \bar{y}), f(x, z) \\
\bar{x}, \quad x
\end{array}\right) \text { and } \varphi^{\prime}\left(\begin{array}{c}
f(\bar{x}, \bar{y}), f(x, z) \\
\bar{y}, z
\end{array}\right), \Gamma_{2}(P) \rightarrow \Theta_{2}(P) \\
{\left[\begin{array}{c}
\operatorname{resp.} \\
\varphi^{\prime}\left(\begin{array}{c}
f(\bar{x}, \bar{y}), f(x, z) \\
\bar{x},
\end{array}\right), \Gamma_{1}(P) \rightarrow \Theta_{1}(P) \quad \text { and } \Gamma_{2}(P) \rightarrow \varphi^{\prime}\left(\begin{array}{c}
f(\bar{x}, \bar{y}), f(x, z) \\
y,
\end{array}\right)
\end{array}\right]}
\end{gathered}
$$

are both provable in $I L(P)$. Take $\varphi=(\exists v) \varphi^{\prime}(f(\bar{x}, \bar{y}), v)$ (resp. $\varphi=(\forall v) \varphi^{\prime}$ $(f(\bar{x}, \bar{y}), v))$. This formula $\varphi$ is the desired one.

Case 5. $R=(\psi)^{*}$ with $\psi$ is of type $(F, G)$. We may assume that $\mathscr{D}$ is of the form:

$$
R \frac{\Gamma \stackrel{\downarrow \mathscr{E}_{1}}{\longrightarrow} \bar{a}=\bar{a}^{\prime} \Gamma \stackrel{\downarrow \mathscr{E}_{2}}{\longrightarrow} \bar{b}=\bar{b}^{\prime} \Gamma \stackrel{\downarrow \mathscr{E}_{3}}{\longrightarrow} F(\bar{a}) \Gamma, G(\bar{b}) \stackrel{\downarrow \mathscr{E}_{4}}{\longrightarrow} \Theta}{\Gamma \longrightarrow \Theta},
$$

where $I\left(\bar{a}^{\prime}, \bar{b}^{\prime}\right) \subseteq \Gamma$ and $\bar{a}, \bar{a}^{\prime}, \bar{b}, \bar{b}^{\prime}$ are sequences of free variables with the same length $n$.

Let $\Delta=\left\langle\bar{x}, \bar{y} ; \Gamma_{1}(P), \Gamma_{2}(Q) ; \Theta_{1}(P), \Theta_{2}(Q)\right\rangle$ be an $I$-partition of $\Gamma \rightarrow \Theta$. Then $\bar{a}^{\prime} \subseteq \bar{x}$ and $\bar{b}^{\prime} \subseteq \bar{y}$. Let $\bar{z}$ be a repetition-free enumeration of the elements of $\bar{a} \cup \bar{b}-\bar{x} \cup \bar{y}$. Then $\Delta_{1}=\left\langle\bar{x} \frown \bar{z}, \bar{y} \frown \bar{z} ; \Gamma_{1}(P), \Gamma_{2}(Q) ;\left\{\bar{a}=\bar{a}^{\prime}\right\}, \emptyset\right\rangle$, 
$\Delta_{2}=\left\langle\bar{x} \frown \bar{z}, \bar{y} \frown \bar{z} ; \Gamma_{1}(P), \Gamma_{2}(Q) ; \emptyset,\left\{\bar{b}=\bar{b}^{\prime}\right\}\right\rangle, \quad \Delta_{3}=\left\langle\bar{x} \frown \bar{z}, \bar{y} \frown \bar{z} ; \Gamma_{1}(P), \Gamma_{2}(Q) ;\right.$ $\{F(\bar{a})\}, \emptyset\rangle, \quad \Delta_{4}=\left\langle\bar{x} \frown \bar{z}, \bar{y} \frown \bar{z} ; \Gamma_{1}(P), \Gamma_{2}(Q) \cup\{G(\bar{b})\} ; \Theta_{1}(P), \Theta_{2}(Q)\right\rangle$ are I-partitions of $S_{1}=\Gamma \rightarrow \bar{a}=\bar{a}^{\prime}, S_{2}=\Gamma \rightarrow \bar{b}=\bar{b}^{\prime}, S_{3}=\Gamma \rightarrow F(\bar{a})$ and $S_{4}=\Gamma, G(\bar{b})$ $\rightarrow \Theta$, respectively. For each $1 \leqslant i \leqslant 4$, by the induction hypothesis on $\mathscr{E}_{i}$ and the end-sequent $S_{i}$, we get a formula $\varphi_{i}$ in $\mathscr{M}(P)$ which satisfies the conditions (1), (2) on $\Delta_{i}$. Hence the following sequents are provable in $I L(P)$.

$$
\begin{array}{cc}
\varphi_{1}(\bar{z}), \Gamma_{1}(P) \longrightarrow \bar{a}=\bar{a}^{\prime}, & \Gamma_{2}(P) \longrightarrow \varphi_{1}^{\prime}(\bar{z}) ; \\
\Gamma_{1}(P) \longrightarrow \varphi_{2}(\bar{z}), & \varphi_{2}^{\prime}(\bar{z}), \Gamma_{2}(P) \longrightarrow \bar{b}=\bar{b}^{\prime} ; \\
\varphi_{3}(\bar{z}), \Gamma_{1}(P) \longrightarrow F(\bar{a}), & \Gamma_{2}(P) \longrightarrow \varphi_{3}^{\prime}(\bar{z}) ;
\end{array}
$$

and if $\Theta_{1}(P)$ is empty (resp, $\Theta_{2}(Q)$ is empty) then

$$
\begin{gathered}
\Gamma_{1}(P) \longrightarrow \varphi_{4}(\bar{z}), \quad \varphi_{4}^{\prime}(\bar{z}), \Gamma_{2}(P), F(\bar{b}) \longrightarrow \Theta_{2}(P) \\
\left(\operatorname{resp.} \varphi_{4}(\bar{z}), \Gamma_{1}(P) \longrightarrow \Theta_{1}(P), \quad \Gamma_{2}(P), F(\bar{b}) \longrightarrow \varphi_{4}^{\prime}(\bar{z})\right. \text { ) }
\end{gathered}
$$

where $\varphi_{i}(\bar{z})=\varphi_{i}\left(\begin{array}{c}f(\bar{x}, \bar{y}), f(\bar{z}, \bar{z}) \\ \bar{x}\end{array}\right)$ and $\varphi_{i}^{\prime}(\bar{z})=\varphi_{i}\left(\begin{array}{c}f(\bar{x}, \bar{y}), f(\bar{z}, \bar{z}) \\ \bar{y}\end{array}\right)$ for each $1 \leqslant i \leqslant 4$. If we take $\varphi$ as the formula obtained from $\varphi_{2} \wedge \varphi_{4} \wedge\left(\varphi_{1} \wedge \varphi_{3}\right.$ . $\left.\supset . F\left(f\left(\bar{a}^{\prime}, \bar{b}^{\prime}\right)\right)\right)$ on binding all members of $f(\bar{z}, \bar{z})$ by the quantifier $\exists$ or $\forall$, then $\varphi$ is our desired formula in $\mathscr{M}(P)$.

Our proof of Proposition is here completed and so our proof of Main theorem.

\section{§4. $T(P)$ does not define $P$ explicitly up to disjunction}

We cannot hope that the condition (1) in the Main theorem and the following condition:

(3) $T(P) \vdash_{I L(P)} \bigvee_{i=1}^{n}(\forall \bar{u})\left(P(\bar{u}) \equiv \varphi_{i}(\bar{u})\right)$ for some formulas $\varphi_{1}(\bar{x}), \cdots$, $\varphi_{n}(\bar{x})$ in $L$

are equivalent.

CountereXample. Let $T(P)=\{(\forall u)(P(u) \equiv(R(u) \supset(\exists v)(P(v) \wedge R(v))))$, where $R$ is a unary predicate in $L$. In this case, condition (1) holds by our Main theorem but condition (3) does not holds as follows. For simplicity, we denote the single element of $T(P)$ by $T(P)$ itself. Assume that

$$
\vdash_{I L(P)} T(P) \longrightarrow \bigvee_{i=1}^{n}(\forall u)\left(P(u) \equiv \varphi_{i}(u)\right),
$$

where $\varphi_{1}(x), \cdots, \varphi_{n}(x)$ are formulas in $L$. Since two sentences 


$$
\begin{aligned}
T(P) \equiv & \forall(u)(P(u) . \supset . R(u) \supset(\exists v)(P(v) \wedge R(v))) \\
& \wedge(\forall u)(R(u) \supset(\exists v)(P(v) \wedge R(v)) . \supset . P(u))
\end{aligned}
$$

and

$$
(\forall u)(P(u) . \supset . R(u) \supset(\exists v)(P(v) \wedge R(v)))
$$

are provable in $I L(P)$, we get

$$
\vdash_{I L(P)}(\forall u)(R(u) \supset(\exists v)(P(v) \wedge R(v)) . \supset . P(u)) \longrightarrow \bigvee_{i=1}^{n}(\forall u)\left(P(u) \equiv \varphi_{i}(u)\right) .
$$

Put $H(P)=(\forall u)(R(u) \supset(\exists v)(P(v) \wedge R(v)) . \supset . P(u))$. Then $H(P)$ is a Harrop formula (see [5]) and so, for some $1 \leqslant i \leqslant n$

$$
\vdash_{I L(P)} H(P) \longrightarrow(\forall u)\left(P(u) \equiv \varphi_{i}(u)\right) .
$$

Let $x$ be a free variable. From $\langle 2\rangle$, the following two sequents are provable in $I L(P)$ :

$$
\begin{aligned}
& H(P), P(x) \longrightarrow \varphi_{i}(x), \\
& H(P), \varphi_{i}(x) \longrightarrow P(x) .
\end{aligned}
$$

Hence we can easily show that $\varphi_{i}(x)$ is provable in $I L(P)$ by replacing all the occurrences of $P(*)$ by $T$ in a derivation with end-sequent $\langle 3\rangle$ and next applying cut rules. Hence, the following sequent is provable in $I L(P)$,

$$
H(P) \longrightarrow(\forall u) P(u) \text {. }
$$

On the other hand, it is obvious that the sequent $\langle 5\rangle$ is unprovable even in the classical logic which includes $P$ as a predicate constant. This is a contradiction. Therefore, our assumption $\langle 1\rangle$ does not hold.

\section{REFERENCES}

[1] S. Kochen, Topics in the theory of definition, Proc. of Model Theory Symposium, Berkeley, 1963 (1965), 170-176.

[2] N. Motohashi, Interpolation theorem and characterization theorem, Ann. Japan Assoc. Philos. Soc., 4 (1972), 85-150.

[ 3 ] - A new theorem on definability in a positive second order logic with countable conjunctions and disjunctions, Proc. Japan Acad., 48 (1972), 153-156.

[4] V. Harnik and M. Makkai, Application of Vaught sentences and the covering theorem, J.S.L., 41, 1 (1976), 171-187.

[5] A. S. Troelstra, Metamathematical Investigation of Intuitionistic Arithmetic and Analysis, Lecture Notes in Mathematics, 344, Springer-Verlag, (1973).

Department of Mathematics

University of Tsukuba

Sakuramura Ibaraki, 300-31

Japan 